\title{
Critical appraisal and role of memantine extended-release in the management of Alzheimer's disease
}

This article was published in the following Dove Press journal:

Degenerative Neurological and Neuromuscular Disease

10 June 2011

Number of times this article has been viewed

\author{
Indrapal Singh \\ George T Grossberg \\ Department of Neurology and \\ Psychiatry, St Louis University School \\ of Medicine, St Louis, MO, USA
}

Correspondence: Indrapal Singh Department of Neurology and Psychiatry, Division of Geriatric Psychiatry, Saint Louis University School of Medicine, 1438 South Grand Blvd, St Louis, MO 63108, USA

Tel + I 3 I4 9774829

Fax + I 3149774878

Email isingh2@slu.edu

\begin{abstract}
Alzheimer's disease (AD) is a progressive, degenerative brain disease. Currently available US FDA-approved pharmacological management options for AD are cholinesterase inhibitors (donepezil, rivastigmine, and galantamine) and the N-methyl-D-aspartate receptor antagonist (memantine). These treatment options may provide symptomatic benefits. Medication adherence is one of the many problems faced by the caregivers of patients with dementia. The currently available FDA-approved memantine immediate-release (IR) has been found safe and efficacious at a dose of $10 \mathrm{mg}$ twice daily ( $20 \mathrm{mg}$ /day) both as monotherapy and in combination with cholinesterase inhibitors in moderate to severe dementia. Memantine extended-release (ER) $28 \mathrm{mg}$, a new once-daily form at a higher dose, has also been found to be safe and well tolerated in 2 studies: one in 24 healthy volunteers which showed relatively minor fluctuation in plasma levels of memantine during the steady-state dosing interval and second, a multinational multi-center study, comparing memantine ER $28 \mathrm{mg}$ and placebo in patients with moderate to severe $\mathrm{AD}$ stable on concurrent cholinesterase inhibitor (ChEI). In this study, memantine ER was found to be an efficacious drug with a good safety and tolerability profile. Both memantine IR $20 \mathrm{mg}$ and memantine ER $28 \mathrm{mg}$ are now FDA-approved for the treatment of moderate to severe $\mathrm{AD}$, either alone, or in combination with a ChEI. Both are efficacious, safe, and well tolerated. Medication adherence with the ER preparation should improve because of once-daily dosing. There is a possibility of superior efficacy of the higher dose memantine ER (28 mg) over the currently approved dosage of memantine IR (20 mg). However, further randomized, controlled, double-blind comparator studies of memantine ER $28 \mathrm{mg}$ vs memantine IR $20 \mathrm{mg}$ and/or $30 \mathrm{mg}$ in single and/or divided doses are required to assess the possible benefits of memantine ER over memantine IR.
\end{abstract}

Keywords: Alzheimer's disease, memantine extended-release, memantine immediate-release, memantine

\section{Introduction}

Dementia is a syndrome that affects memory, thinking, behavior, and ability to perform daily activities. Alzheimer's disease (AD) is the most common cause of dementia. It is a progressive, irreversible degenerative disease of the brain. An estimated 35.6 million people are living with dementia worldwide in 2010. This number is estimated to nearly double every 20 years, to 65.7 million in 2030 and to 115.4 million in $2050 .{ }^{1}$ Currently there is no curative treatment for AD. However persistent antidementia treatment (cholinesterase inhibitors and/or memantine) has a positive impact on AD progression assessed by multiple cognitive, functional, and global outcome measures. ${ }^{2}$ There is significant burden of care on caregivers as the disease progresses specifically in the moderate to severe stages of AD. Medication adherence is one of the many 
problems faced by the caregivers. The total number of medications taken per day, including antidementia medications, is inversely related to adherence. Simpler, less frequent dosing regimens result in better adherence as well as less burden on caregivers. ${ }^{3}$

Current US Food and Drug Administration (FDA)approved pharmacological treatments for AD include cholinesterase inhibitors (donepezil, rivastigmine, and galantamine) and the N-methyl-D-aspartate (NMDA) receptor antagonist (memantine). Memantine is FDA-approved for the treatment of moderate to severe Alzheimer's disease either as monotherapy or in combination with a cholinesterase inhibitor. ${ }^{4-6}$ Memantine is a safe and effective drug both as monotherapy and in combination with cholinesterase inhibitors (ChEI) in moderate to severe dementia. ${ }^{7}$ Both short- and long-term memantine treatment of patients with AD is safe and well tolerated, with an adverse event profile almost similar to that of placebo. ${ }^{8}$ In addition, due to its low potential for drug-drug interactions, memantine may be particularly well suited for combination therapy. ${ }^{8}$ The current FDA-approved memantine dosage is $10 \mathrm{mg}$ twice daily (20 mg/day), in the form of an immediate-release (IR) tablet or solution. Studies for safety, tolerability, and efficacy have been done with these dosages and preparations. In this article, we examine the current form and dosage relative to safety, tolerability, and efficacy of memantine IR, $10 \mathrm{mg}$ twice daily, as well as memantine extended-release (ER) $28 \mathrm{mg}$, a new once-daily form and dosage of the medication which has been approved by the FDA and is expected to be available soon for clinical usage.

\section{Mechanism of action}

It is hypothesized that glutamate-mediated neurotoxicity is involved in the pathogenesis of AD. ${ }^{9}$ The presence of beta amyloid stimulates excessive glutamate production and the high concentrations of glutamate cause an excess calcium influx through the ion channel that leads to excitotoxicity and eventual cell death. ${ }^{9,10}$ The excitotoxicity is mediated through NMDA receptors via increased selective permeability to calcium. Memantine is a noncompetitive NMDA receptor antagonist as well as a partial agonist of the NMDA receptor with low to moderate affinity. At lower concentrations, memantine continues to allow the physiological synaptic signal to be recognized, while at a higher concentration, it blocks the pathological effect of excessive glutaminergic stimulation. This mechanism may allow memantine to act as a neuroprotective agent against excitotoxicity as well as an enhancer of synaptic integrity. ${ }^{11}$

\section{Pharmacokinetic profile of memantine IR vs memantine ER}

Memantine is well absorbed orally. Food has no significant effect on its rate of absorption. The majority of ingested memantine IR is excreted via urine. The hepatic microsomal cytochrome P450 system has no significant role in the metabolism of memantine IR and patients with mild to moderate renal disease do not require any change in dosage. ${ }^{12}$ However, daily dose reduction of memantine is recommended to $10 \mathrm{mg}$ daily in severe renal disease. ${ }^{12,13}$ At present, the recommended daily dose of memantine IR is $10 \mathrm{mg}$ twice daily administered in the form of IR tablets or solution. Memantine IR is eliminated in a mono-exponential manner with terminal elimination half-life of 60 to 100 hours, indicating that memantine may be given in a $20-\mathrm{mg}$ once-daily regimen. ${ }^{14}$

A new ER form, which contains a higher dose of memantine ( $28 \mathrm{mg}$ ) has been developed to improve adherence and possibly efficacy. ${ }^{6,8,13}$ The absorption rate of memantine ER $28 \mathrm{mg}$ is slow, with a median time to $\mathrm{T}_{\max }$ of 12 hours compared with 3 to 7 hours observed with the immediate release formulation. ${ }^{15}$ The mean elimination half-life $\left(\mathrm{T}_{1 / 2}\right)$ of memantine ER $28 \mathrm{mg}(55.7 \pm 9.5)$ is quite similar to that of the immediate-release formulation (44 to 61 hours). ${ }^{15-17}$ There is minimal fluctuation in plasma levels of memantine ER during steady state dosing intervals. The $\mathrm{C}_{\max }$ at steady state of memantine ER in plasma was $127.1 \pm 21.1 \mathrm{ng} / \mathrm{mL}$ which is approximately 3 to 4 times the $\mathrm{C}_{\max }$ reported for memantine $20 \mathrm{mg}$ (22 to $46 \mathrm{ng} / \mathrm{mL}$ ). ${ }^{16,18}$ However, there was no direct relationship between the increased $\mathrm{C}_{\text {max }}$ in the memantine ER study and increased adverse events. ${ }^{16}$

\section{Memantine IR: once-daily vs twice-daily dosage}

A 12-week randomized, double-blind multicenter study in the UK investigated 3 dosing schedules of memantine: OD1 (20 mg once daily with a 1-step up-titration); OD3 (20 mg once daily with a 3-step uptitration); and BID3 (10 mg twice daily with a 3 -step up-titration as currently recommended) ${ }^{19}$ The study comprised 78 patients with moderate to severe AD (DSM-IV-TR criteria; Mini-Mental State Examination [MMSE] score $\leq 18$ ), $70 \%$ of whom were on stable dosing of cholinesterase inhibitors (ChEI) initiated $\geq 3$ months before study initiation. Safety and tolerability were assessed by the number of drop-outs, adverse events (AEs) and monitoring of vital signs. The number of drop-outs was low: 3 of 27 in OD1, 1 of 25 in OD3, and 2 of 26 in BID3. One or more AEs were reported in 9 patients in OD1, 7 patients in OD3, and 
12 patients in BID3. Most AEs were mild or moderate, and typical for the population studied; no clinically important differences in AEs or vital signs were observed between the different dosing schedules. There were no between-group differences in efficacy, as assessed by clinical global severity and clinical global change. These results are consistent with the good safety profile of memantine observed in larger studies. Although relatively small in size, the study indicates that once-daily dosing and twice-daily dosing of memantine are similar in terms of safety and tolerability.

This extension study was conducted as a multicenter randomized double-blind, parallel group, 8-week phase (Phase A), followed by a 20-week open-level (Phase B). This study was done with the aim of evaluating the long term safety of memantine in patients with mild to moderate dementia, and of investigating the tolerability of once daily dose administration. ${ }^{20}$ This 28 -week extension study enrolled 314 patients with mild to moderate AD (MMSE score 10 to 22) who had completed a 24 -week, double-blind, placebo controlled lead-in clinical trial of memantine in AD. ${ }^{20}$ During phase A, patients who had received placebo in the lead-in study were titrated to memantine $20 \mathrm{mg}$ with either twice-daily dosing or with once-daily dosing. Patients who had received active drug in the lead-in study continued to receive $20 \mathrm{mg}$ /day memantine, and were either maintained on twice-daily dosing or switched to once-daily dosing. Following an 8-week randomized double-blind dose titration phase (Phase A: used to assess the tolerability of different dosing regimens), subjects were assigned to continuous open label memantine (10 mg, twice a day) treatment for 20 weeks (Phase B). Safety outcome measures included treatment-emergent adverse events (AEs), deaths, vital signs, electrocardiograms, and laboratory parameters. During the 28 -week study (Phase A + Phase B), the most common AEs were falls and other injuries (both $10.8 \%$ ). AEs resulted in treatment discontinuation in $6.7 \%$ of patients. Discontinuations due to AEs were similar in the oncedaily dosing groups compared with the twice daily dosing groups. During dose titration, completion rates were greater than $90 \%$ for both groups. Conversion to once-daily dosing in patients already receiving twice daily doses of memantine was also well tolerated. Memantine monotherapy in patients with mild to moderate $\mathrm{AD}$ was safe and well tolerated for at least 1 year. Once-daily dosing during titration and short-term maintenance therapy was also safe and well tolerated.

\section{Memantine ER 28 mg}

The first study related to ER memantine was conducted by Periclou and $\mathrm{Hu}$, whose stated objective was to investigate the safety, tolerability, and pharmacokinetics of an ER memantine capsule $(28 \mathrm{mg})$ in 24 healthy volunteers. ${ }^{16}$ This was a phase I single center open label study in which 16 men and 8 women received a single daily oral dose of memantine ER for 29 days, as follows: $7 \mathrm{mg}$ (days 1-3), $14 \mathrm{mg}$ (days 4-9), $21 \mathrm{mg}$ (days 10-15), and $28 \mathrm{mg}$ (days 16-29). Safety and tolerability were assessed throughout the study, for each individual who received at least one dose of the drug. Blood samples were obtained on days 1, 27, 28, and 29, and analyzed for memantine using liquid chromatography coupled to tandem mass spectrometry. There were no serious adverse events (AEs) or deaths. Four participants discontinued the study: one due to an $\mathrm{AE}$ (somnolence), one due to a protocol violation, and two withdrew consent. Treatment-emergent AEs were all mild in severity and in most cases judged possibly related to study drug. The most common AEs were headache, somnolence, and dizziness. One subject had an increase in aspartate and alanine aminotransferases which normalized after discontinuation of study drug. No one had a potentially clinically significant (PCS) change in vital signs from screening to endpoint, or a PCS change during the treatment, relative to pre-dose values.

It was found that the absorption rate of memantine in the ER capsule preparation was relatively slow, with a median $T_{\text {max }}$ of 12 hours compared with 3 to 7 hours with the immediate-release formulation. ${ }^{15,16} \mathrm{Mean}_{\mathrm{C}_{\max }}$ and $\mathrm{C}_{\min }$ were $127.1 \pm 21.1 \mathrm{ng} / \mathrm{mL}$ and $102.5 \pm 16.5 \mathrm{ng} / \mathrm{mL}$, respectively. Mean elimination halflife was $55.7 \pm 9.5$ hours and area under the concentration-time curve from 0-24 hours $\left(\mathrm{AUC}_{0-24}\right)$ was $2726 \pm 430 \mathrm{ng} / \mathrm{mL}$. In this study, memantine ER capsule administration was found to be safe and well-tolerated. The relatively minor difference between $\mathrm{C}_{\max }$ and $\mathrm{C}_{\min }$ suggests only minor fluctuations in plasma levels during the steady-state dosing interval.

The first and only multicenter study comparing memantine ER $28 \mathrm{mg}$ and placebo was done in patients with moderate to severe AD (MMSE range: 3 to 14) who were receiving concurrent, stable dose of cholinesterase inhibitor (ChEI) therapy for 3 months or more. ${ }^{21}$ This multinational, randomized, double-blind, placebo-controlled, parallel group design study included participants who were at least 50 years of age with a diagnosis of probable Alzheimer's disease by DSM-IV-TR (Diagnostic and Statistical Manual of Mental Disorders - Text Revision) or NINCDS-ADRDA (National Institute of Neurological and Communicative Disorders and Stroke and the Alzheimer's Disease and Related Disorders Association) criteria, supported by MRI or CT scan with MMSE scores of 3-14. Patients with other Axis I disorders, other organic brain diseases, or predominant delusions, a Modified Hachinski Ischemia Score $>4$ at screening, treatment 
with more than one $\mathrm{ChEI}$, memantine taken within 1 month of screening or inability to perform at least 1 item on the Severe Impairment Battery (SIB) at baseline were excluded. Patients with clinically significant or active pulmonary, gastrointestinal, renal, hepatic, endocrine, or cardiovascular system disease were also excluded. All the included patients first completed a 2-week, single-blind, placebo-only period, followed by a 24-week, double-blind period of treatment with memantine ER capsule or placebo. Primary outcome measures were the Severe Impairment Battery (SIB) and the Clinician's Interview Based Impression of Change-Plus (CIBIC-Plus). Primary efficacy parameters were changes from baseline to week 24 in SIB total score and the CIBIC-Plus rating using the last observation carried forward approach. The SIB data were analyzed using analysis of covariance, with treatment group and study center as the factors and baseline values as the covariate. The CIBIC-Plus data were analyzed using the Cochran-MantelHaenszel test with modified ridit scores, controlling for study center. Secondary and additional efficacy measures included the Alzheimer's Disease Cooperative Study-Activities of Daily Living 19-item (ADCS-ADL19), Neuropsychiatric Inventory (NPI), and the Verbal (Semantic) Fluency Test. Safety and tolerability were evaluated by physical exam, ECG, vital signs, blood, urine analysis, and reported adverse effects.

A total of 677 patients were enrolled for the study. Of these (mean MMSE score: 10.8), 342 and 335 patients were randomized to receive once-daily memantine ER capsule $(28 \mathrm{mg})$ and placebo respectively, with a completion rate of $79.8 \%$ and $81.2 \%$. Memantine ER dose was titrated in weekly increments of $7 \mathrm{mg}$, reaching a target dose of $28 \mathrm{mg} /$ day at the beginning of week 4 . At week 24, patients treated with memantine ER and ChEI showed a statistically significant improvement over placebo/ChEI-treated patients on both the SIB $(P=0.001)$ and CIBIC-Plus $(P=0.008)$. Memantine ER was well-tolerated. In both groups, most AEs were mild or moderate in severity. The most common AEs occurring in the memantine ER/ChEI group at a higher rate relative to the placebo/ChEI group were headache (5.6\% vs 5.1\%), diarrhea (5.0\% vs $3.9 \%)$, and dizziness (4.7\% vs $1.5 \%)$. Four memantine-treated and 5 placebo-treated patients died during this trial; no death was judged related to memantine treatment. Patients treated with memantine ER $28 \mathrm{mg} / \mathrm{ChEI}$ also showed statistically significant benefits on outcomes of behavior as measured by the NPI $(P=0.005)$, and on the verbal fluency test $(P=0.004)$ compared with patients who were receiving placebo/ChEI. Activities of daily living, as assessed by the ADCS-ADL19, were not statistically improved compared with placebo at the end of the trial. Memantine ER capsule (28 mg), given once daily was found to be an effective, safe, and well-tolerated therapy for patients with moderate to severe $\mathrm{AD}$, receiving concurrent, stable ChEI treatment. Based on this study, the FDA approved memantine ER $28 \mathrm{mg}$ for the treatment of moderate to severe AD.

\section{Discussion}

Memantine ER $28 \mathrm{mg}$ may have two possible benefits over memantine IR: once-daily dosage, and the possible superior benefit of a higher dose. However, the study by Jones et al indicates that once-daily dosing of memantine IR $20 \mathrm{mg}$ is equivalent to twice-daily dosing of memantine IR $10 \mathrm{mg}$ in terms of safety and tolerability. ${ }^{19}$ The expected benefit of the memantine ER preparation in comparison to memantine IR once-daily dosing may have even better tolerability secondary to less plasma level fluctuation during steady state dosing interval. However, a head-to-head comparative study of memantine ER $28 \mathrm{mg}$ vs memantine IR at equivalent higher dosages with once-daily dosing is required to assess the benefits of the ER preparation vs the IR preparation. A 'once-daily regimen' of memantine ER may be better than the package insert recommended 'twice-daily regimen' of the IR preparation relative to patient adherence. A 'once-daily regimen' may also help reduce the stress on caregivers.

The phase III study of memantine ER $28 \mathrm{mg}$ was done in patients with moderate to severe Alzheimer's disease, who were on a stable dose of a ChEI. ${ }^{21}$ The patients treated with memantine ER/ChEI showed a statistically significant improvement over placebo/ChEI-treated patients. However, this significant improvement may represent primarily an effect of 'combination therapy' of a ChEI and memantine ER rather than the effects of memantine ER alone. ${ }^{21}$ A comparative study of this higher dose memantine ER $28 \mathrm{mg}$ vs combination therapy of memantine ER/ChEI would give us more information relative to the benefits of combination therapy with memantine ER $28 \mathrm{mg}$. There is also a possibility that memantine ER $28 \mathrm{mg}$ may have superior efficacy over memantine IR $20 \mathrm{mg}$ because of dose dependent effects in cognitive, global, and behavioral measures. However the possibility of dose-dependent superior efficacy can be commented on definitely only after a randomized controlled trial comparing the efficacy of memantine ER $28 \mathrm{mg}$ /day and memantine IR $20 \mathrm{mg} /$ day in moderate to severe Alzheimer's disease.

It is recommended that the daily dosage of memantine IR should be reduced to $10 \mathrm{mg}$ in patients with severe renal impairment. ${ }^{12}$ However, no information is available on the dosing, safety, and tolerability of memantine ER $28 \mathrm{mg}$ in patients with renal disease; further studies are needed in this regard, specifically in patients with severe renal impairment. Since memantine ER $28 \mathrm{mg}$ has been found to be safe, well 
tolerated, and effective in a once daily, convenient dose, this may be the next step in the management of moderate to severe Alzheimer's disease, for which treatment options available are still limited. ${ }^{16,21}$ Memantine ER $28 \mathrm{mg}$ should be available on the market for clinical use in the next few months. Increased clinical use of memantine ER $28 \mathrm{mg}$ in patients with moderate to severe Alzheimer's disease will give us more information about this drug. Further randomized, controlled, double-blind comparator studies of memantine ER $28 \mathrm{mg}$ vs memantine IR $20 \mathrm{mg}$ to assess the effectiveness, and memantine ER $28 \mathrm{mg}$ vs memantine IR at an equivalent dose $(30 \mathrm{mg})$ to assess comparative efficacy, tolerability, and safety are required for more definite answers.

\section{Conclusion}

Both memantine IR $20 \mathrm{mg}$ and memantine ER $28 \mathrm{mg}$ are now FDA-approved for the treatment of moderate to severe AD, either alone, or in combination with a ChEI. Both are safe and well tolerated. Reliable, once-daily dosing with the ER preparation should improve medication adherence.

Unanswered is the question: is memantine ER $28 \mathrm{mg}$ therapeutically superior to memantine IR $20 \mathrm{mg}$ ? We cannot answer this question until a head-to-head study is done. Until such a time, memantine ER $28 \mathrm{mg}$ may be most useful for patients who do not respond to the IR $20 \mathrm{mg}$ dose or who lose response to the $20 \mathrm{mg}$ dose.

\section{Disclosure}

The authors declare no conflicts of interest in this work.

\section{References}

1. World Alzheimer Report 2009, Executive Summary. http://www.alz co.uk/research/files/WorldAlzheimerReport-ExecutiveSummary.pdf. Accessed January 15, 2011.

2. Rountree SD, Chan W, Pavlik VN, Darby EJ, Siddiqui S, Doody RS. Persistent treatment with cholinesterase inhibitors and/or memantine slows clinical progression of Alzheimer disease. Alzheimers Res Ther. 2009;1:7.

3. Claxton AJ, Cramer J, Pierce C. A systematic review of the associations between dose regimens and medication compliance. Clin Ther. 2001; 23:1296-1310.

4. Tariot PN, Farlow MR, Grossberg GT. Memantine treatment in patients with moderate to severe Alzheimer disease already receiving donepezil: a randomized controlled trial. JAMA. 2004;291: $317-324$.
5. Reisberg B, Doody R, Stoffler A. Memantine in moderate-to-severe Alzheimer's disease. N Engl J Med. 2003;348:1333-1341.

6. McShane R, Areosa Sastre A, Minakaran N. Memantine for dementia. Cochrane Database Syst Rev. 2006;2:CD003154.

7. Thomas S, Grossberg GT. Memantine: a review of studies into its safety and efficacy in treating Alzheimer's disease and other dementias. Clin Interv Aging. 2009;4:367-377.

8. Farlow MR, Graham SM, Gustavo, Alva G. Memantine for the treatment of Alzheimer's disease: tolerability and safety data from clinical trials Drug Saf. 2008;31:577-585.

9. Whetsell WOJ, Shapira NA. Neuroexcitation, excitotoxicity and human neurological disease. Lab Invest. 1993;68:372-387.

10. Hynd MR, Scott HL, Dodd PR. Glutamate-mediated excitotoxicity and neurodegeneration in Alzheimer's disease. Neurochem Int. 2004;45: 583-595.

11. Danysz W, Parsons CG. The NMDA receptor antagonist memantine as a symptomatological and neuroprotective treatment for Alzheimer's disease: preclinical evidence. Int J Geriatr Psychiatry. 2003;18: S23-S32.

12. Periclou A, Ventura D, Rao N, Abramowitz W. Pharmacokinetic study of memantine in healthy and renally impaired subjects. Clin Pharmacol Ther. 2006;79:134-143.

13. Bassil Nhttp://informahealthcare.com/action/show Popup? citid=citart $1 \&$ id=end-a $1 \&$ doi $=10.1517 / 14656566.2010 .493874$, Thaipisuttikul Phttp://informahealthcare.com/action/showPopup? citid=citart $1 \&$ id=end-a $\&$ doi $=10.1517 / 14656566.2010 .493874$, Grossberg GT, Memantine ER, a once-daily formulation for the treatment of Alzheimer's disease. Expert Opin Pharmacother. 2010; 11:1765-1771.

14. Kornhuber J, Kennepohl EM, Bleich S. Memantine pharmacotherapy: a naturalistic study using a population pharmacokinetic approach. Clin Pharmacokinet. 2007;46:599-612.

15. Namenda ${ }^{\circledR}$ : US Prescribing Information. St. Louis, MO: Forest Pharmaceuticals, Inc., April 2007.

16. Periclou A, Hu Y. Extended-release memantine capsule $(28 \mathrm{mg}$, once daily): a multiple dose, open-label study evaluating steady-state pharmacokinetics in healthy volunteers [abstract from poster presentation]. Presented at the 11th International Conference on Alzheimer's Disease, 26-31 July 2008; Chicago, IL, USA.

17. Chládek J, Žaludek B, Sova P, Franc A, Šišpera L, Martínková. Steady-state bioequivalence studies of two memantine tablet and oral solution formulations in healthy volunteers. J Appl Biomed. 2008;6:39-45.

18. Van Marum RJ. Update on the use of memantine in Alzheimer's disease. Neuropsychiatr Dis Treat. 2009;5:237-247.

19. Jones RW, Bayer A, Inglis F, Barker A, Phul R. Safety and tolerability of once-daily versus twice-daily memantine: a randomised, doubleblind study in moderate to severe Alzheimer's disease. Int $J$ Geriatr Psychiatry. 2007;22:258-262.

20. Ott BR, Blake LM, Kagan E, Resnick M. Open label, multicenter, 28-week extension study of the safety and tolerability of memantine in patients with mild to moderate Alzheimer's disease. J Neurol. 2007; 254:351-358.

21. Grossberg GT, Manes F, Allegri R. A multinational, randomized, doubleblind, placebo-controlled, parallel-group trial of memantine extendedrelease capsule ( $28 \mathrm{mg}$, once daily) in patients with moderate to severe Alzheimer's disease [abstract from poster presentation]. Presented at the 11th International Conference on Alzheimer's Disease, 26-1 July 2008; Chicago, IL, USA.
Degenerative Neurological and Neuromuscular Disease

\section{Publish your work in this journal}

Degenerative Neurological and Neuromuscular Disease is an international, peer-reviewed, open access journal focusing on research into degenerative neurological and neuromuscular disease, identification of therapeutic targets and the optimal use of preventative and integrated treatment interventions to achieve improved outcomes, enhanced

\section{Dovepress}

survival and quality of life for the patient. The manuscript management system is completely online and includes a very quick and fair peer-review system. Visit http://www.dovepress.com/testimonials.php to read real quotes from published authors. 